\title{
Targeting EZH2 for the treatment of soft tissue sarcomas
}

\author{
Magdalena Karolak', Ian Tracy ${ }^{1}$, Janet Shipley ${ }^{2}$, Zoë S Walters ${ }^{1}$ \\ ${ }^{1}$ Translational Epigenomics Team, Cancer Sciences, Faculty of Medicine, University of Southampton, Southampton SO16 6YD, \\ UK. \\ ${ }^{2}$ Sarcoma Molecular Pathology Team, Divisions of Molecular Pathology and Cancer Therapeutics, The Institute of Cancer \\ Research, London SM2 5NG, UK.
}

Correspondence to: Zoë S Walters, Translational Epigenomics Team, Cancer Sciences, Faculty of Medicine, University of Southampton, Southampton SO166YD, UK. E-mail: z.s.walters@soton.ac.uk

How to cite this article: Karolak M, Tracy I, Shipley J, Walters ZS. Targeting EZH2 for the treatment of soft tissue sarcomas. J Cancer Metastasis Treat 2021;7:15. https://dx.doi.org/10.20517/2394-4722.2021.05

Received: 7 Jan 2021 First Decision: 2 Feb 2021 Revised: 8 Feb 2021 Accepted: 25 Feb 2021 Available online: 26 Mar 2021

Academic Editor: lan Judson Copy Editor: Yue-Yue Zhang Production Editor: Yue-Yue Zhang

\begin{abstract}
Soft tissue sarcomas (STS) are a heterogenous group of rare malignancies of mesenchymal origin, affecting both children and adults. The majority of STS have a poor prognosis and advanced stage at the time of diagnosis. Standard treatments for STS largely constitute tumour resection with chemotherapy and/or radiotherapy, and there has been little significant advancement in the application of novel therapies for treatment of these tumours. The current multimodal approach to therapy often leads to long-term side effects, and for some patients, resistance to cytotoxic agents is associated with local recurrence and/or metastasis. There is, therefore, a need for novel therapeutic strategies for the treatment of STS. Recent advances in epigenetics have implicated the histone methyltransferase, EZH2, in the development and progression of diseases such as breast cancer, lymphoma and more recently STS. Here we will review the current literature for EZH2 in STS, including high expression of EZH2 in STS and correlation of this with specific features of malignancy (metastasis, histological grade, and prognosis). The effects of targeting EZH2 using RNA interference and small molecule inhibitors will also be reviewed and the potential for the use of EZH2 inhibition in therapeutic strategies for STS patients will be discussed.
\end{abstract}

Keywords: EZH2, epigenetics, therapy 


\section{INTRODUCTION}

Soft tissue sarcomas (STS) constitute a highly heterogenous group of rare malignancies believed to be of mesenchymal origin. Mesenchymal stem cells harbour powerful self-renewal capacity and multi-lineage differentiation potential into various tissues, such as adipose, muscle, bone and cartilage. Over 60 distinct STS subtypes have been identified with regard to their biological features and clinical manifestation, making the classification of these tumours often problematic and challenging. STS can occur in any part of the body, but the most frequent sites include the extremities, head and neck areas as well as trunk. STS comprise around $1 \%$ of all adult cancers and around $8 \%$ of all paediatric and adolescent tumours. However, the spectrum of STS subtypes differs in these different age ranges. Despite being relatively rare cancers, the vast majority of diagnosed cases of STS present with highly aggressive behaviour, poor prognosis and advanced stage of the disease at point of detection. Many STS can be thought of as undifferentiated tumours, where STS cells express early markers of lineage-specific differentiation but do not differentiate into the mature, benign tissue type. For example, rhabdomyosarcomas (RMS) resemble immature skeletal muscle cells that have failed to complete differentiation and cell cycle arrest, and the restoration of this process is considered to be of therapeutic value $e^{[1,2]}$.

A number of STS discussed in this review, harbour specific genetic hallmarks, such as chromosome translocations and specific gene mutations or amplification events. These contribute to the process of oncogenesis and may be useful diagnostic features. An example is the recurrent chromosomal translocation $\mathrm{t}(\mathrm{X} ; 18)(\mathrm{p} 11.2 ; \mathrm{q} 11.12)$ found in synovial sarcomas (SS), leading to gene fusions between SS18 on chromosome 18 and either $S_{S X}$, SSX2 or in rare cases $S S X_{4}$ on chromosome $\mathrm{X}$. The abnormal fusion protein SS18-SSX disrupts the epigenetic regulation of gene expression and is believed to drive sarcoma formation in $\mathrm{SS}^{[3,4]}$. Another example is malignant peripheral nerve sheath tumours (MPNSTs), in which Neurofibromin (NF1) tumour suppressor gene mutations are thought to drive malignancy in some patients. Such mutations lead to inactivation of NF1 protein and therefore to development and pathogenesis of MPNSTs $^{[5]}$. The same holds true for extremely rare and aggressive atypical teratoid rhabdoid tumours (ATRTs, brain) or malignant rhabdoid tumours (MRTs, kidneys and soft tissues) where homozygous inactivation of SMARCB1, and resultant deficiency of SMARCB1 protein, a member of the SWI/SNF complex, occur in the majority of these malignancies ${ }^{[6]}$. RMS, ATRT and MRT tumours affect predominantly infants and/or children/adolescents, with RMS being the most frequent, accounting for 50\% of all STS in childhood ${ }^{[2,7]}$.

Current treatment of STS is largely based on tumour resection followed by chemotherapy and/or radiotherapy. This multimodal approach often leads to long-term side effects and, due to resistance to cytotoxic agents in large proportion of sarcoma patients, it also results in local recurrence as well as metastasis. Despite intensification of treatment regimes, little significant advancement in treatment outcomes for high-risk patients with these malignancies has been noted in recent years ${ }^{[8]}$. The low efficiency/failure of the standard therapies in STS highlights a fundamental necessity for development of novel, more effective and less harmful treatment strategies for sarcoma patients.

\section{ROLE OF EZH2 IN STS}

Enhancer of Zeste Homologue 2 (EZH2) is the catalytic subunit of Polycomb Repressive Complex 2 (PRC2), a multiprotein complex comprised of four core units, EED, SUZ12, RbBP4, and EZH2, although a number of other auxiliary proteins have also been shown to modulate PRC2 activity [Figure 1] ${ }^{[9]}$. PRC2 is crucial for maintaining the epigenetic state of the cells through modulation of chromatin structure and by such means, regulation of gene expression. $\mathrm{EZH} 2$ is a histone methyltransferase whose mode of action is observed as addition of methyl groups at lysine 27 in histone $\mathrm{H} 3$ through its active SET domain motif, 


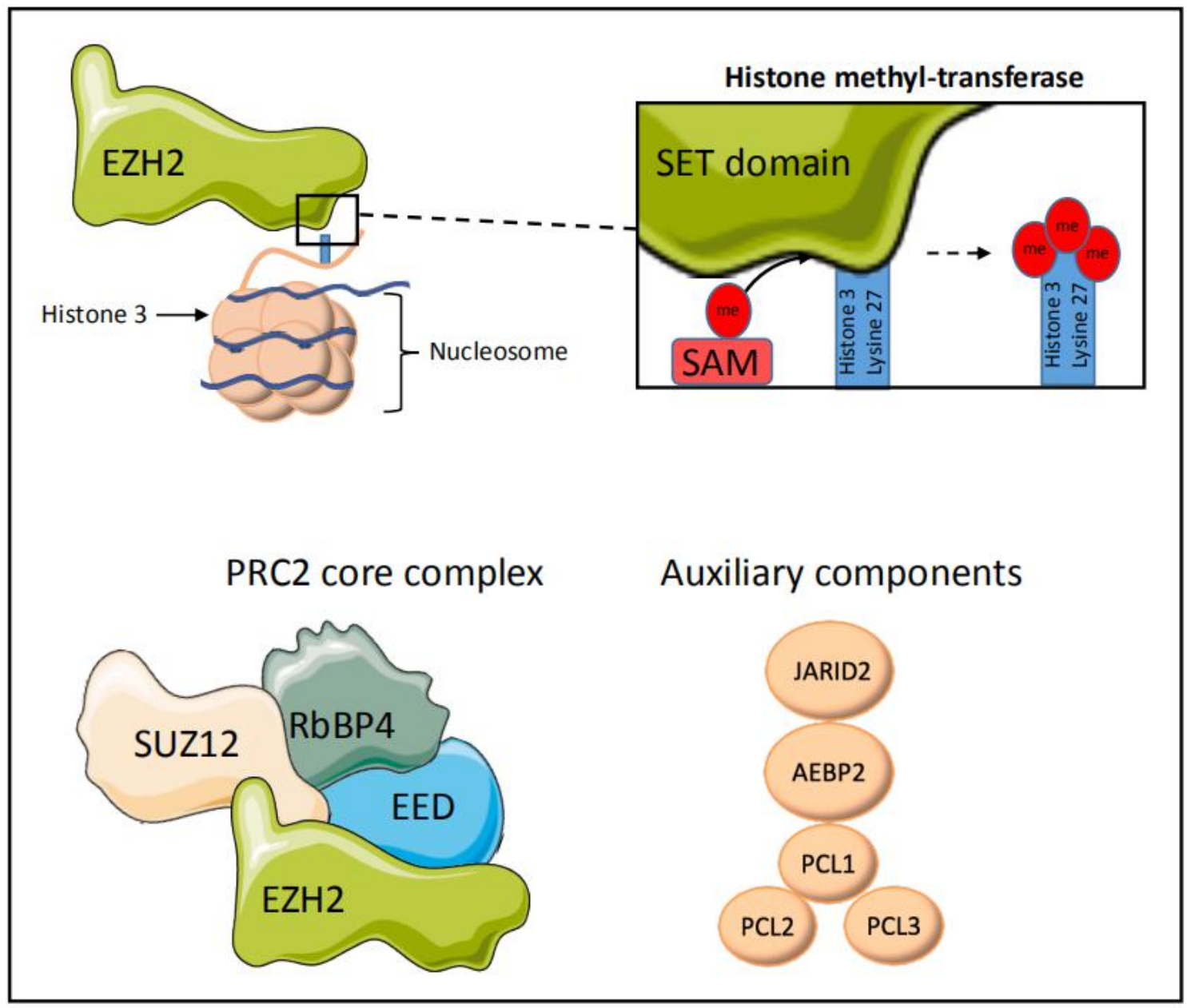

Figure 1. EZH2 is a histone methyltransferase, catalysing the transfer of donor methyl groups from SAM to H3K27 via its C-terminal SET domain. EZH2 predominantly functions as part of a multi-protein complex, PRC2, containing the core units SUZ12, EED, and RbBP4, which aid in recognition and targeting of EZH2 activity to histone 3 lysine-27. A number of other auxiliary proteins are known to complex with PRC2 but are disposable to its core activity. All structures are representative only. Subset of images from Servier medical art https://smart.servier.com/?s=nucleosome. SAM: S-adenosyl-L-methione.

resulting in the formation of the $\mathrm{H} 3 \mathrm{~K} 27 \mathrm{me} 3$ epigenetic mark and subsequent transcriptional repression ${ }^{[10]}$. $\mathrm{EZH} 2 / \mathrm{PRC} 2$-facilitated methylation of $\mathrm{H}_{3} \mathrm{~K} 27$ is part of a gene expression regulatory process, and the end effect of this chain of events is the compaction of chromatin from "open" euchromatin to "closed" heterochromatin and functional repression of gene expression by preventing the binding of nuclear transcription factors [Figure 2]. Many of the genes which EZH2 is involved in silencing are effectors of cellular differentiation ${ }^{[11]}$. Thus, EZH2 activity is broadly associated with the prevention of terminal differentiation and lineage commitment in cells, and maintaining the capacity for self-renewal and a pluripotent stem cell phenotype in those cells with its high expression ${ }^{[12]}$. Through its role in gene expression regulation, EZH2 is involved in a variety of biological processes, including regulation of cell cycle, cell differentiation, cell proliferation, division, and senescence.

A recent meta-analysis of 8 studies has shown that mutations in $E Z H 2$ confer a poorer prognosis for patients with myeloid neoplasms, such as acute myeloid leukaemia ${ }^{[13]}$. Likewise results from a Phase-II trial of the EZH2 inhibitor, Tazemetostat, in relapsed or refractory follicular lymphoma patients with $\mathrm{EZH} 2$ 


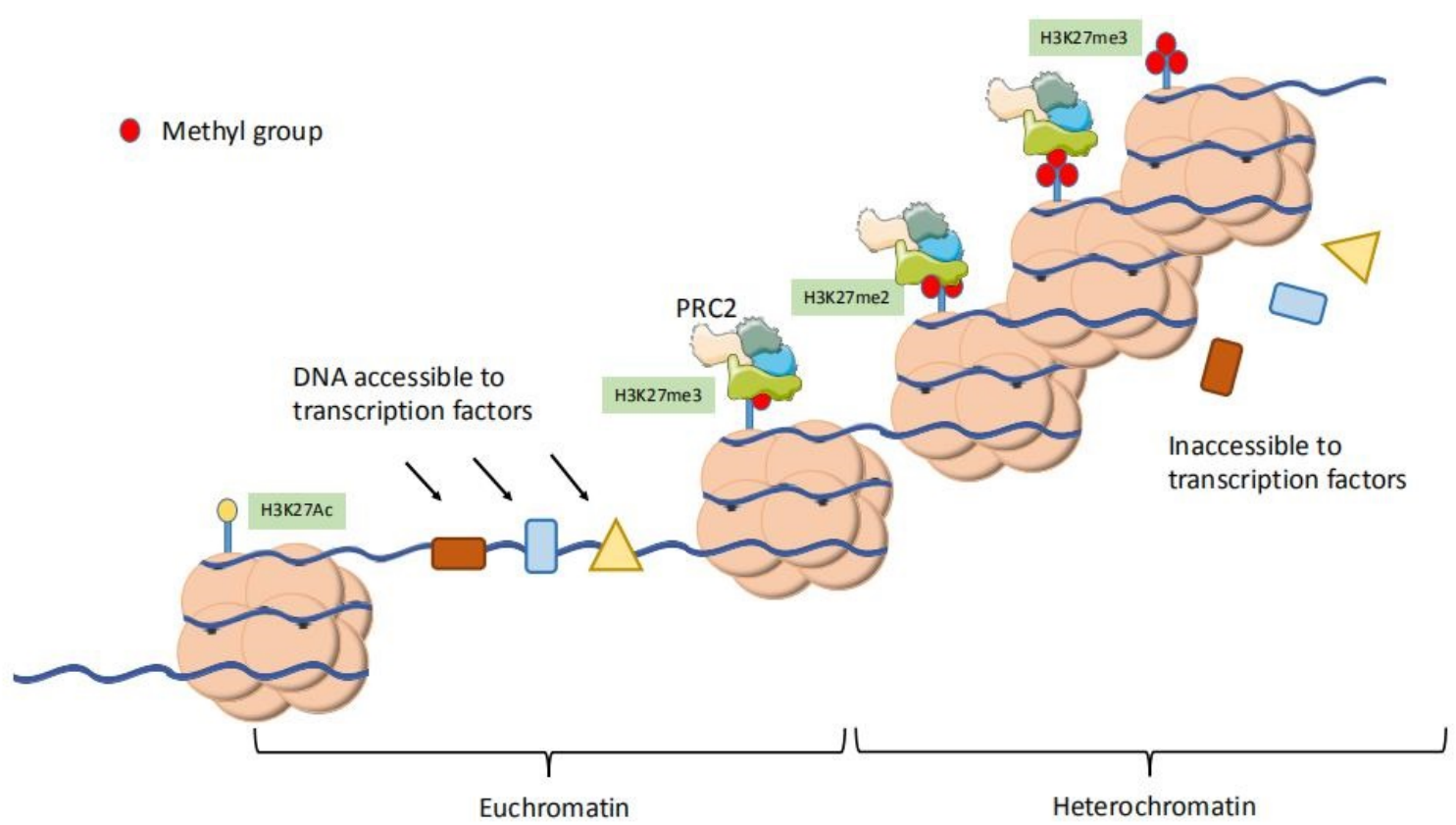

Figure 2. Histone methyltransferase activity of EZH2/PRC2 is part of a sequence of epigenetic alterations that lead to condensation of chromatin into tightly bound structures, thereby physically preventing access to transcription factors and other transcriptional machineries.

mutations have promising initial results ${ }^{[14]}$. However, mutations of EZH2 are not prevalent in soft tissue sarcomas. A study in 2012 screened a range of sarcoma types for mutations in a selection of seven genes, including $E Z H 2$, known to be recurrently mutated in non-sarcomatous cancers. Of these genes only two PIK3CA mutations and one JAK mutation were observed in the entire cohort of samples $(3 / 108 ; 2.8 \%)$. The soft tissue sarcomas investigated in that study included malignant fibrous histiocytomas, rhabdomyosarcomas, malignant peripheral nerve sheath tumours, leiomyosarcomas, synovial sarcomas, liposarcomas, angiosarcomas, and Ewing sarcomas ${ }^{[15]}$.

Rather, EZH2 is overexpressed in many STS in comparison to normal tissue, which may be attributed to the cell of origin of these tumours. STS are thought to be derived from mesenchymal stem cells, where the balance of EZH2 expression along with other histone modifying enzymes is thought to control the differentiation lineage process ${ }^{[16-19]}$. The reason why overexpression of wildtype $\mathrm{EZH} 2$ leads to associated poor outcomes can primarily be understood in terms of the normal function of EZH2. EZH2 is involved in silencing genes which drive differentiation in developing cells/tissues ${ }^{[20]}$. Under normal development, EZH2 expression fades over time, to become almost undetectable in adult specialized cells and tissues [Figure 3 ]$^{[10]}$. The inappropriately timed expression of $\mathrm{EZH} 2$ is therefore thought to lead to an undifferentiated phenotype with cells maintaining the ability for self-renewal.

Of increasing importance and focus are interactions between PRC2 and SWItch/Sucrose Non-Fermentable (SWI/SNF) complex. The latter is another chromatin remodelling machinery with an opposed function to $\mathrm{PRC}^{[10]}$. The antagonistic mode of action of both complexes is essential to ensuring homeostasis and proper epigenetic profile of the cells. Mutations in the SWI/SNF complex subunit SMARCB1 (also referred to as INI1) result in loss of SMARCB1 regulatory function, leading to the dysregulation of EZH2 activity and thus abnormal gene repression and oncogenic capacity of $\mathrm{EZH} 2^{[21-23]}$. As a consequence, it is observed as development and progression of solid tumours ${ }^{[24]}$. Nonetheless, the EZH2 aberration linked to SWI/SNF 


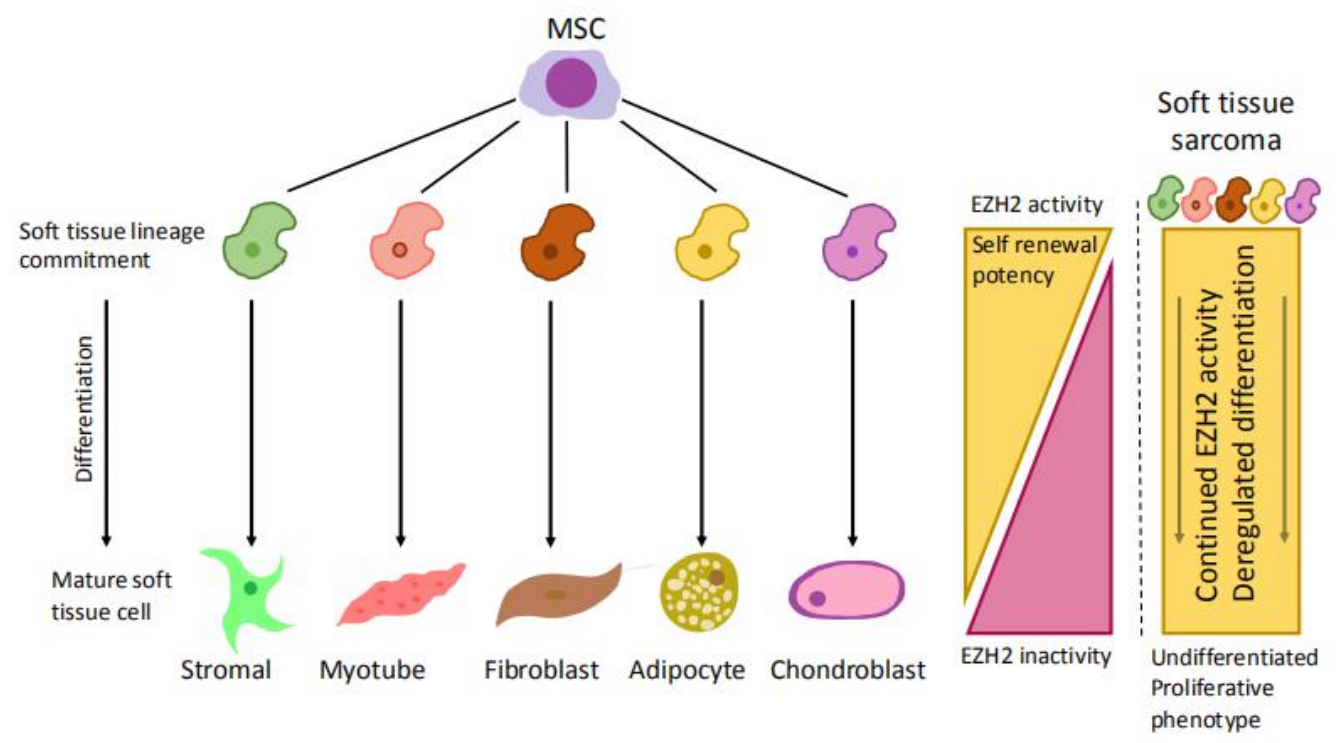

Figure 3. Soft tissue sarcomas (STS) are thought to be derived from lineage committed mesenchymal stem cells. Aberrant EZH2 expression and/or activity is believed to prevent normal terminal differentiation, leading to continued proliferation and poorly differentiated phenotypes in STS.

constitutes only one out of many other causes of EZH2 dysregulation and subsequent pathogenesis ${ }^{[25]}$.

\section{CORRELATIONS OF EZH2 WITH CLINICAL FEATURES}

\section{EZH2 upregulation}

Higher levels of EZH2 have been noted in MPNST tumours and MPNST cell lines, compared to normal human Schwann cells ${ }^{[5]}$. The same phenomena were detected in RMS tumour samples and cell lines, with little or no $\mathrm{EZH}_{2}$ expression observed in normal skeletal muscle tissue ${ }^{[26]}$, benign rhabdomyoma or tumouradjacent skeletal muscle ${ }^{[20]}$. This is also true for leiomyosarcoma, where EZH2 exhibited elevated levels with high sensitivity and specificity in malignant tissues in contrast to benign leiomyoma or normal myometrium $^{[20]}$. Another study investigating the expression of EZH2 in embryonal (ERMS) and alveolar (ARMS) RMS cell lines and patient samples showed similar findings demonstrating high upregulation of the RNA in comparison to myoblasts as a control ${ }^{[27]}$.

\section{Prognosis}

In STS prognosis is usually poor, dependent on other factors such as stage of malignancy and tumour size at time of detection, the patient's age, localisation of the tumour and presence/ absence of metastatic lesions. In the prognosis of SS, high levels of EZH2 protein are associated with worse clinical outcome, especially in the poorly differentiated subtype ${ }^{[28]}$. In a study including 104 patient-derived tumour samples (with 27 having prior chemotherapy, 25 radiotherapy and 13 both) of mixed STS: SS, leiomyosarcomas, RMS, epithelioid sarcomas, malignant fibrous histiocytomas, myxofibrosarcomas and liposarcomas, higher levels of EZH2 protein were independently correlated with unfavourable prognosis for each STS type ${ }^{[29]}$.

\section{Metastasis}

In studies comprising 55 SS primary tumour samples without preoperative chemo- or radiotherapy ${ }^{[28]}$, and 29 SS tumour samples (22/29 treated with chemotherapy and radiation following surgery ${ }^{[30]}$, higher levels of $\mathrm{EZH} 2$ and $\mathrm{H}_{3} \mathrm{~K} 27 \mathrm{me} 3$ as examined by immunohistochemistry (IHC) were observed in patients where distant metastasis has occurred ${ }^{[28,30]}$. EZH2 was also significantly associated with distant metastasis in other 
sarcoma subtypes, such as LMS, ES, LS, RMS and $\mathrm{MFH}^{[29]}$. In a study conducted on 17 paediatric RMS and extraosseous Ewing sarcoma (EES) tumour samples, elevated EZH2 protein levels were associated with increased aggressiveness of the disease and the presence of metastasis to lymph nodes and/or distant parts of the body at the time of diagnosis ${ }^{[31]}$.

\section{Survival}

Higher EZH2 expression in 14 patients with SS was significantly associated with worse survival. However, the same was not found to be significant in 31 liposarcoma and $36 \mathrm{MFH}$ patients ${ }^{[29]}$. In a study conducted on 105 sarcoma tumour samples from patients with no prior adjuvant therapy (28 RMS, 15 Ewing's Sarcoma, 30 osteosarcoma, 18 SS, 14 epithelioid sarcoma and chondrosarcoma), higher EZH2 expression and higher $\mathrm{H} 3 \mathrm{~K} 27 \mathrm{me} 3$ levels were associated with significantly shortened overall survival ${ }^{[32]}$. However, the inclusion of tumour types other than STS means that this may therefore not represent the true OS for these types of malignancy. Expression of EZH2 was also a marker of lower likelihood of survival in paediatric samples of 11 RMS and 6 EES tumours, compared to the EZH2-negative cases ${ }^{[31]}$. Furthermore, 14 out of 29 SS patients with lower EZH2 levels, had a significantly prolonged overall survival than those of higher EZH2 expression profiles $^{[30]}$.

\section{Histological grade and clinical stage}

In a study using 104 patient-derived tumour samples, it was observed that higher EZH2 expression was associated with higher histological grade of the tumour ${ }^{[29]}$. This is inconsistent with another study, in which EZH2 high expression did not show significant correlation with respect to histological grade ${ }^{[30]}$. This may be due to the smaller sample size (29 SS cases). In another paper, consisting of 50 patients-derived SS tumour samples, those with II and III clinical stages had significantly higher EZH2 scores by immunohistochemistry (IHC) than those with stage I with no EZH2 expression found in one case with stage $\mathrm{IV}^{[3]}$. In 17 patients with RMS and EES, high intra-tumour EZH2 scores were present in stage III or IV of the malignancy ${ }^{[31]}$. These inequalities may emerge due to the limited sample sizes, so further investigations are needed.

\section{EZH2 and Ki-67}

In SS EZH2 expression was correlated with Ki-67 scores, especially in poorly differentiated subtypes ${ }^{[28]}$. Abundant expression of Ki-67 with strong positive correlation to EZH2 scores was also observed in other types of STS, such as LMS, alveolar and embryonal RMS ${ }^{[33]}$, LS, ES and $\mathrm{MFH}^{[29]}$. These clearly indicated the positive association between EZH2 expression and proliferative potential in soft tissue malignancies. In another study including various subtypes of RMS, such as ARMS, ERMS, unclassified, spindle cell and pleomorphic RMS, the Ki-67 and EZH2 were also parallel in all primary and recurrent RMS tumour samples $^{[34]}$.

\section{TARGETING EZH2 IN STS \\ EZH2 modulation through RNA interference}

\section{Rhabdomyosarcoma}

Knockdown of EZH2 through vector-based shRNAs in the embryonal RMS cell line RD resulted in induction of differentiation due to the increase of muscle specific factors, restored recruitment of multiprotein complexes at muscle-specific promoters and activated expression of MyoD, as well as its binding abilities, crucial for myogenesis ${ }^{[1]}$. Another study showed that depletion of EZH2 with siRNAs in the $\mathrm{RD}$ cell line led to inhibition of cell proliferation and significant decrease of H3K27me3. These were followed by the expression of proteins specific to terminal myogenic differentiation ${ }^{[35,36]}$, indicating that the ablation of $E Z H 2$ restores myogenic differentiation ability of embryonal RMS cells. Alveolar RMS cell lines (RH30 and $\mathrm{RH} 4$, containing PAX3-FOXO1 fusion gene), which underwent $E Z \mathrm{H}_{2}$ interference through siRNA or shRNA manifested inhibition of cellular proliferation, migration, anchorage-independent growth 
and reduction of cell survival ${ }^{[36-38]}$. Surprisingly, the EZH2 depletion led to expression of pro-apoptotic genes instead of differentiation genes, resulting in initiation of cellular death ${ }^{[37]}$. The authors demonstrate that the mechanism of apoptosis in these cells is likely due to derepression of pro-apoptotic gene including the tumour suppressor FBXO32 after EZH2 knockdown, as well as in reduction of myogenic proteins such as Myogenin and MyoD, indicating an induction of apoptosis over differentiation ${ }^{[37]}$. A separate study also demonstrated apoptosis in RH30 cells after siRNA silencing of EZH2. However, they also showed that modest reduction of EZH2 in both ERMS (RD) and ARMS (RH30) cells led to differentiation and expression of the terminal differentiation marker myosin heavy chain (MHC) in the presence of differentiation conditions ${ }^{[36]}$. It is worth noting that $\mathrm{EZH} 2$ is involved in a feedback loop with other regulatory components such as miR-101, which may serve as a tumour suppressor, and through repression of this molecule is able to promote tumorigenesis in ERMS, as was shown in a study conducted by Vella and colleagues $^{[2]}$. Silencing of EZH2 through siRNAs in ERMS cell lines increased the expression of several miRNAs such as miR-29b, miR-214 and miR-101. The latter caused down-regulation of both mRNA and protein levels of EZH2 and thus, reduction of the tumorigenic potential of ERMS cells in vitro ${ }^{[2]}$.

\section{Rhabdoid Tumours (MRTs and ATRTs)}

Silencing of EZH2 by shRNAs in SMARCB1-deficient paediatric ATRT cell lines (BT12, BT16 and patientderived UPN 737) has been shown to reduce cell proliferation and concomitantly induce cellular senescence and apoptosis, with the biggest effects seen with combination EZH2-silencing by shRNA and administration of DZNep. It was also demonstrated that inhibition of EZH2 affected crucial pathways and molecules involved in cell cycle regulation with downregulation of $\mathrm{E} 2 \mathrm{~F}$ and $\mathrm{c}-\mathrm{Myc}^{[39]}$. Furthermore, in an MRT cell line model, sustained knockdown of EZH2 through siRNA manifested as reduction of cellular growth and SMARCB1 restoration ${ }^{[40]}$.

\section{Other STS Subtypes (MPNST, SS, LS)}

Stable knockdown of EZH2 using shRNAs in both non-NF1 related and NF-1 related MPNST cell lines resulted in increased apoptosis, inhibited growth and decreased viability of malignant cells in vitro. By using MPNST immunodeficient mouse xenografts it was demonstrated that injected EZH2-targeted shRNAs were also able to inhibit tumorigenicity in vivo ${ }^{[5]}$. In the SS cell lines possessing either SS18-SSX1 (Aska-SS, Yamato-SS) or SS18-SSX2 translocation (Fuji, SYO-1) the silencing of EZH2 by siRNA and shRNA led to dose-dependent inhibition of cell proliferation in all four cell lines and decreased levels of $\mathrm{H} 3 \mathrm{~K} 27 \mathrm{me}^{[3]}$. Conversely, depletion of EZH2 through RNAi in well-differentiated and dedifferentiated liposarcoma cell lines with addition of steroids and insulin, led to cell proliferation inhibition, followed by induction of differentiation in vitro $^{[41]}$.

\section{EZH2 modulation through use of small molecular inhibitors}

Recently, EZH2 has been the focus of numerous drug discovery efforts, resulting in a number of tool compounds and clinical candidates targeting EZH2 being developed. The first to be developed was 3deazaneplanocin A (DZNep), whose mechanism of action is as an S-adenosyl-L-homocysteine (SAH) hydrolase inhibitor. DZNep represses S-adenosyl-L-methionine-dependent histone methyltransferase activity and thus is not a specific EZH2 inhibitor. A number of S-adenosyl-methionine competitive EZH2specific inhibitors have since been developed [Table 1], a number of which have been taken into clinical trials for blood cancers and solid tumours (Tazemetostat and GSK126) ${ }^{[1,42-45]}$.

\section{Synovial sarcoma}

In SS18-SSX1 (HS-SY-II) and SS18-SSX2 (Fuji) translocation-positive and SMARCB1-deficient synovial sarcoma cell lines, treatment with tazemetostat led to a concentration-dependent inhibition of cell growth and apoptosis in vitro. Administration of tazemetostat or EPZ011989 in xenograft models of SS, carrying 
Table 1. Table of the EZH2 inhibitors discussed in the review, along with their specificity over EZH2, effects on the protein and mechanisms of action.

\begin{tabular}{llll}
\hline EZH2 Inhibitor & Specificity for EZH2 & Effect on EZH2 & ${\text { Mode of } \text { Action }^{[46]}}^{\text {E }}$ \\
\hline DZNep (3-deazaneplanocin A) & Non-specific & EZH2 degradation & Inhibition of SAH hydrolase with methyltransferase activity \\
EPZ011989 & Specific & EZH2 catalytic inhibition & SAM-competitive inhibition of PRC2 \\
EPZ005687 & Specific & EZH2 catalytic inhibition & SAM-competitive inhibition of PRC2 \\
GSK126 & Specific & EZH2 catalytic inhibition & SAM-competitive inhibition of PRC2 \\
GSK343 & Specific & EZH2 catalytic inhibition & SAM-competitive inhibition of PRC2 \\
MC1945/ MC1948 & Specific & EZH2 catalytic inhibition & SAM-competitive inhibition of PRC2 \\
UNC1999 & Specific & EZH2 catalytic inhibition & SAM-competitive inhibition of PRC2 \\
Tazemetostat (EPZ-6438) & Specific & EZH2 catalytic inhibition & SAM-competitive inhibition of PRC2 \\
\hline
\end{tabular}

DZNep is the least specific inhibitor, leading to measurable reduction in global levels of EZH2, whilst the others act mostly as catalytic inhibitors.

either the HS-SY-II/Fuji cell line or patient-derived tumour, resulted in a dose-dependent decrease in tumour volume and inhibition of tumour growth, followed by decreased $\mathrm{H} 3 \mathrm{~K} 27 \mathrm{me} 3$ in vivo. Combined therapy of tazemetostat and the chemotherapeutic drug doxorubicin demonstrated remarkable antitumor activity in Fuji xenografts, relative to each monotherapy alone in the course of treatment. However, after discontinuance of dosing, tumour regrowth was observed. The same treatment regimen in HS-SY-II xenografts did not show the same effects, and instead dose-dependent reduction of intra-tumoral H3K27me3 levels was seen, which was attributed to additional genetic aberrations in this cell line. In patient-derived xenografts, two out of three models exhibited significant inhibition of tumour growth after administration of tazemetostat, most markedly in xenografts with highest SMARCB1 deficiency ${ }^{[4]}$. This suggests the SS18-SSX translocation-positive SS cells are sensitive for treatment with tazemetostat. Dosage of another EZH2 inhibitor, EPZ005687 in SS18-SSX translocation-positive four cell lines (Aska-SS, SYO-1, Fuji, Yamato-SS) resulted in decreased expression of $\mathrm{H}_{3} \mathrm{~K} 27 \mathrm{me} 3$, and dose-dependent inhibition of cell proliferation and migration (up to $48 \mathrm{hrs}$ after $72 \mathrm{hrs}$ of dosing). However, the combined treatment of EPZ005687 with three chemotherapeutic drugs (etopside/topotecan/doxorubicin) in Aska-SS and SYO-1 cell lines did not show significant synergy ${ }^{[3]}$. In a phase 2 clinical trial involving 33 SS patients with confirmed either SMARCB1 loss/depletion or SS18-SSX translocation and median of 2 preceding systemic treatments, single agent tazemetostat treatment resulted in stable disease in 11 patients with only 5 of those having stable disease lasting 16 weeks or more. No objective responses in pre-treated patients were seen ${ }^{[47]}$.

\section{Rhabdomyosarcoma}

A study comprising ERMS RD cell line and administration of a differentiating agent 12-Otetradecanoylphorbol-13-acetate (TPA) in combination with GSK126 led to markedly more differentiation (assessed by observation of morphological changes in cell phenotype) than the use of either drugs alone, coupled with an increase in MHC expression. Dosage of the drugs after differentiation-induction led to a reduction in cell growth ${ }^{[48]}$. The treatment with DZNep or two EZH2 inhibitors, MC1948 and the more potent MC1945, in the ERMS RD cell line showed dose-dependent inhibition of the RMS cells proliferation and reduction of $\mathrm{H}_{3} \mathrm{~K} 27 \mathrm{me} 3$. Furthermore, immunofluorescence analysis for MHC revealed signs of myotube-like structures, suggesting restoration of myogenic differentiation in vitro. The same treatment regimen with MC1945 applied to mouse xenograft resulted in reduction of tumour growth and induction of differentiation in vivo, which confirms anti-tumour and pro-differentiative activity of these inhibitors in both, cell lines and living organism ${ }^{[35,38]}$. Similarly, administration of either DZNep or MC1945 to ARMS cell lines significantly affected their proliferative potential and led to manifestation of pro-apoptotic cell features, mirroring the RNAi data. The same results were obtained in vivo, demonstrating reduction of tumour volume and depletion of EZH2 and Ki-67-positive cells in a mouse xenograft ${ }^{[3738]}$. Notably, EZH2 
inhibition in ARMS led to apoptosis, whereas in ERMS resulted in induction of differentiation. The possible interpretation of these may be that both subtypes originate from the same cellular lineage but undergo distinct differentiation pathways and generate specific clinical phenotypes ${ }^{[49]}$, which determines their response to the therapy.

A more recent paper describes a new treatment approach involving dual "hybrid" EZH2/HDAC inhibitor in RH4 cell line. This strategy led to cell cycle arrest, apoptotic events, affected cell viability, enhanced differentiation and reduction of $\mathrm{H} 3 \mathrm{~K} 27 \mathrm{me} 3$. The higher doses of the dual drug were more efficient and worked in an intensified manner ${ }^{[50]}$, which confirms the more complex or combined treatment in STS at certain conditions works better than monotherapy. Accordingly, the concurrent administration of TPA/GSK126 and Vincristine to chemoresistant and undifferentiated MYOD1+/NOG+ ERMS cells led to enhanced effectiveness of the therapy and increased synergy. The administration of TPA/GSK126 at first showed growing levels of MYOG protein, indicating cell differentiation. Following treatment with vincristine, higher decreases in cell viability and reduction of surviving RMS cells were observed, suggesting drugs synergy ${ }^{[51]}$. Administration of DZNep to ERMS cells led to restoration of several micro-RNAs, such as miR-29b, miR-214 and miR-101. The same results were obtained through forced expression of a mature miR-101 precursor, leading to restoration of miR-101 levels, followed by down-regulation of both mRNA and protein levels of EZH2 and therefore, reduction of the tumorigenic potential of ERMS cells in vitro. These discoveries confirm the miR-101 is directly targeted by EZH2 and lower levels of this microRNA may contribute to EZH2 overexpression and hence, promote cancer progression in $\mathrm{ERMS}^{[2]}$. In contrast to these findings, the Paediatric Preclinical Testing Program (PPTP) study demonstrated that administration of the highly potent and EZH2-selective inhibitor tazemetostat as a single agent in ARMS mouse xenografts did not show promising antitumour activity ${ }^{[52]}$.

\section{Malignant rhabdoid tumours}

The effectiveness of tazemetostat has also been tested against rhabdoid tumours in vivo by the PPTP. Tazemetostat was administered to immunodeficient mouse MRT xenografts, demonstrating significant antitumor activity and differences observed in event free survival (EFS) in 5 out of 7 rhabdoid xenografts, compared to non-rhabdoid models. Both, MRT and ATRT xenografts in which the EZH2 inhibition was the most effective, were identified as SMARCB1-deficient ${ }^{[52]}$. In another study, administration of EPZ-6438 in MRT cells led to a dose-dependent decrease in $\mathrm{H} 3 \mathrm{~K} 27 \mathrm{me}$, with other histone epigenetic marks being unaffected. Furthermore, proliferation of cancerous cells was significantly reduced compared to control SMARCB1 wild-type cells. EPZ-6438 treatment in immunocompromised mouse xenografts bearing G401 cells showed completed elimination of the tumour without recurrence after termination of dosing. Smaller doses of the drug resulted in stable tumour growth at first, followed by delay in growth ${ }^{[6]}$. Another approach evaluating DZNep in combination with conventional cytostatic drugs (doxorubicin/etoposide) or epigenetic agents (5-Aza-CdR/SAHA) to three, anatomically distinct MRT cell lines (kidney, brain, and liver) displayed significantly synergy. Addition of DZNep led to remarkable intensification of anti-proliferative outcome in vitro ${ }^{[7]}$, except for treatment with doxorubicin, in which addition of DZNep did not show significant differences ${ }^{[53]}$. In another study, treatment of G401 cells with GSK126, GSK343 or UNC1999 showed reduction of $\mathrm{H} 3 \mathrm{~K} 27 \mathrm{me} 3$ levels and only modest inhibition of cell proliferation. Furthermore, the combination of a histone deacetylase inhibitor, Panobinostat, with GSK126 resulted in a significant reduction in EZH2 expression, and anti-proliferative and pro-differentiation effects in vitro and in vivo, compared to use of a single agent ${ }^{[40]}$, as demonstrated in other tumour types ${ }^{[4-57]}$.

\section{Atypical teratoid rhabdoid tumours}

In ATRTs, use of DZNep resulted in anti-proliferative and pro-apoptotic effects, increased sensitivity of cancerous cells to radiation and decreased ability to self-renewal and to form tumour spheres ${ }^{[39]}$. Another 
study showed that cell viability was significantly impaired in vitro, but administration of DZNep in vivo did not change the survival time relative to control or systemic-treated/combination-treated xenografts ${ }^{[5]}$. By contrast, in the PPTP study, administration of tazemetostat to immunodeficient mouse ATRT xenografts led to significant differences in EFS, compared to control ${ }^{[52]}$. This suggests that $\mathrm{EZH} 2$-specific inhibition as a single agent is unlikely to be a credible therapeutic option for these patients. Another combination therapy to be tested in ATRT was GSK126 with JQ1 (a bromodomain inhibitor). This resulted in enhanced inhibition of cell proliferation and invasion in vitro, suppressed tumour growth and extended survival in mouse xenografts, compared to the use of each drug alone ${ }^{[59]}$.

\section{Epithelioid sarcoma}

A study involving patient-derived INI1-negative ES tumour samples xenotransplanted to immunodeficient mice and administration of EPZ011989 resulted in tumour growth stabilization at first, followed by tumour volume inhibition of up to $89 \%$ and decreased $\mathrm{H} 3 \mathrm{~K}_{2} 7 \mathrm{me} 3^{[60,61]}$. A clinical trial of patients with SMARCB1(INI1)-negative ES treated with tazemetostat showed similar results to SS patients. Of 62 treated participants, with a median of 1 prior therapy, $15 \%$ exhibited partial responses with objective response rate and disease control rate of $15 \%$ and $26 \%$, respectively. The duration of response to the treatment ranged from 7.1 to 103.0 weeks and a median overall survival of 82.4 weeks was observed for all 62 patients ${ }^{[6]]}$.

\section{Other STS Subtypes (MPNST, LMS, LS)}

A study involving NF1-mutant (S462) and non-NF-1 mutated MPNST (MPNST724) cell lines treated with DZNep demonstrated dose-dependent apoptosis of the cells in vitro relative to untreated normal human Schwann cells. The viability of the cells, measured by the MTT assay, was significantly reduced from 100\% to 30\% and 50\% in S462 and MPNST724 cell lines, respectively. Cell cycle profiles were also altered, indicating cell cycle arrest and inhibition of cell proliferation. Administration of DZNep in immunodeficient mouse xenograft models led to significant reduction of tumour volume, especially at higher doses. Subsequent immunohistochemical analyses revealed inhibition of cell proliferation and induction of apoptosis in vivo ${ }^{[62]}$.

In leiomyosarcoma cell lines, treatment with EPZ011989 resulted in a noticeable decrease in H3K27me3 levels compared to control. Furthermore, in BEZ235-resistant cells prior treatment with EPZ011989 significantly increased their sensitivity to BEZ235 (dactolisib, a dual PI3K/mTOR inhibitor) and a reduction of cancer stem cells (CSCs). In xenografts, pre-treatment with EPZ001989 in combination with BEZ235 led to a significant reduction in tumour growth compared to either drug alone $e^{[63]}$.

Treatment of well-differentiated and dedifferentiated liposarcoma cell lines with GSK343 led to reduction of cell proliferation, followed by a decrease in levels of $\mathrm{H} 3 \mathrm{~K}_{2} 7 \mathrm{me}^{[41]}$. Similarly, combination therapy using GSK126 and 5-aza-dC, a chemical analog of cytidine, in a dedifferentiated cell line showed anti-proliferative effects with a subsequent increased apoptosis and enhanced expression of adipocytes-specific differentiation genes $^{[64]}$. These findings suggest combining epigenetic drugs may be more effective than single agents alone.

A number of clinical trials involving STS patients treated with small molecule inhibitors of EZH2 are currently ongoing. These include treatment with tazemetostat of patients with SS, ES or SMARCB1deficient tumours (NCT02601937, NCT02875548 and NCT02601950). The combined approach of tazemetostat and doxorubicin is also being tested in a clinical trial for patients with advanced ES (NCT04204941). 


\section{THE MECHANISM OF ACTION OF EZH2 IN STS}

It must be emphasized that $\mathrm{EZH} 2$ is a ubiquitous and multifaceted enzyme, involved in numerous regulatory axis and molecular networks, including those promoting oncogenesis. Vella and colleagues demonstrated in ERMS that the levels of a number of miRNAs were restored as EZH2 levels decreased, resulting in anti-cancer activity. Furthermore, depletion or inhibition of EZH2 was highly correlated with altered cell cycle and reduction of expression of several proteins, such as Ki-67 or TBX3 in RMS. However, the role of $\mathrm{EZH} 2$ should be considered differently depending on the cellular context: it can serve as a transcriptional suppressor and a transcriptional co-activator ${ }^{[6,66]}$. Previous research has shown that EZH2 may act in noncanonical manner, independently of PRC2 and its histone methyltransferase function, activating the downstream genes through non-histone targets methylation or direct binding to proteins in several cancer types ${ }^{[67-70]}$. Additionally, the PRC2-SWI/SNF interactions are essential in the regulation of gene expression in several signalling pathways, such as RB, Cyclin D1, MYC and hedgehog, which are impaired in many cancer types ${ }^{[6]}$. In the presented results, EZH2 depletion led to decreased activity of the oncogenic molecules such as $\mathrm{E} 2 \mathrm{~F}$ and c-Myc involved in signal transduction pathways ${ }^{[39]}$, proposing indirect implications of $\mathrm{EZH} 2$ to oncogenesis. When interpreting the outcomes, there is also a need for contextualizing these with relation to the tumour microenvironment (TME), which consists not only of cancer cells but also of extracellular matrix, fibroblasts, adipocytes, endothelia, and immunomodulators such as T and NK cells, tumour-associated macrophages and dendritic cells. All of these play an essential role in the overall response to the therapy and contribute to tumour resistance ${ }^{[7] 1}$. Furthermore, the diversity and number of reactions within the TME may promote cancer escape from immune vigilance ${ }^{[72]}$. Having said this, the complexity of TME may be one of the main reasons for the diverse results among different STS and even within the same histology. Another likely explanation may be heterogenous character of STS, various kinetics and attributes of the cell lines or xenografts, specificity of the inhibitor used and underlying genetic alterations.

\section{The role of PRC2/EZH2 in the immune response - a potential combination therapy?}

The involvement of $\mathrm{PRC} 2 / \mathrm{EZH} 2$ in the immune response is a burgeoning area of research that has implications for both immune-based therapy efficacy and use of EZH2 inhibition as a treatment itself. Immune therapy is not yet a common modality in STS; however, combination therapies utilising EZH2 inhibition and immune therapies may prove to be an effective treatment for STS.

PRC2/EZH2 has been shown to be involved in normal haematopoiesis, the regulation of immune cells, and is essential for T-cell proliferation and anti-tumour immunity ${ }^{\left[{ }^{[3-76]}\right.}$. Conversely it is also implicated in some haematological malignancies ${ }^{[7,78]}$. One potential complication of using EZH2 inhibitors as single agents is unintended effects upon the immune environment. A paper by Huang and colleagues in 2019 identified that in immune-competent mice treated with GSK126, the anti-tumour effects of EZH2 inhibition were reduced compared to immune-deficient mice ${ }^{[79]}$. Importantly the authors also described increased production of myeloid derived suppressor cells (MDSCs) in the GSK126 treated, immune-competent mice, potentially leading to an immune suppressive microenvironment. The authors suggest this as a mechanism behind some of the poorer trial results seen with GSK126.

On the other hand, there is evidence that inhibition of EZH2 may help to improve anti-tumour responses to specific immune check point inhibitors. For example, CPI1205 has been shown to improve the efficacy of the anti-CTLA4 treatment, ipilimumab in a mouse model, primarily by compensating for an increased level of EZH2 induced by ipilimumab itself. The addition of CPI1205 was found to modulate cytotoxic T cells and phenotypically alter Tregs into effector-like T cells and improve the immune response ${ }^{[80]}$. Furthermore, Xiao et al. (2019) ${ }^{[81]}$ showed that in vitro EZH2 negatively regulated the expression of both PD-L1 (CD274) 
and the transcription factor, interferon regulatory factor 1 in hepatocellular carcinoma (HCC) cells and clinical samples. The authors posited that an EZH2 inhibitor may therefore improve responses to anti-PD1/PD-L1 therapy.

In short, EZH2 as a treatment target will certainly be of use in immune therapies; however, due to its role in both normal and abnormal immune processes it is likely that its efficacy will be sub-type/genotype dependant, highlighting the need for studies into this potential combination for the treatment of STS.

\section{CONCLUSIONS}

This review has evaluated EZH2 as a potential target in treatment of STS, an exceptionally diverse group of malignancies, with various underlying genetic and epigenetic alterations defining their development, clinical behaviour and responsiveness to therapies. However, one limitation of this review is that many STS have yet to be studied in the context of EZH2. There are over 60 distinct histological subtypes of STS, for many of which we have not yet understood the contribution of $\mathrm{EZH} 2$ or how it may be exploited for therapeutic benefit. Table 2 summarises the main findings of this review.

STS reviewed in this study almost ubiquitously express high levels of EZH2. As there is no evidence of genetic aberration of EZH2 that might be contributing to this overexpression, it is highly likely that this is the result of the undifferentiated nature of STS, whereby STS cells retain hallmarks of their likely cell of origin, a mesenchymal stem cell. For certain STS, the genetic hallmark of SMI/SNF deregulation, exemplified by SMARCB1 deficiency seen in SS, is likely contributing to the aberrant activity of PRC2 driven by EZH2, which in turn is thought to predispose tumour cells to sensitivity to EZH2 inhibition ${ }^{[52]}$. Indeed, in SS the majority of the cases exhibit lack of SMARCB1 protein as well as the presence of SS18-SSX translocation, which is thought to make cells more prone to EZH2 inhibition.

The correlation of EZH2 protein expression with clinical features of STS is not consistent across studies. However, the most common trend seen includes highly enhanced levels of protein in malignant tumour/cell lines and metastatic lesions, as compared to benign tumour or healthy tissue. The highest divergence arose among EZH2 overexpression with regard to histological grade and clinical stage of the tumours, suggesting either limited sample size or patient/tumour specific EZH2 expression in each phase of the disease.

This study has highlighted both similarities and differences in EZH2 expression and response to EZH2 modulation across STS subtypes. Each STS type has a differing cell lineage type, leading to differing genetic and epigenetic diversity both between STS types and even within STS subtypes. As a consequence, this may explain differences in response to $\mathrm{EZH} 2$ modulation as subset of genes that are both targeting and are targeted by $\mathrm{PRC} 2 / \mathrm{EZH} 2$ are likely to be typical to that cell type. This may therefore also have implications in the use of EZH2 inhibitors as therapeutics and thus differing combinations with $\mathrm{EZH} 2$ inhibitors need to be investigated for different STS.

The results of pharmacological and genetic inhibition of EZH2 seem to be highly promising as a therapeutic option for STS. One key aspect is inhibitor specificity. DZNep is the least specific for EZH2 as its mode of action is inhibition of S-adenosylhomocysteine hydrolase and therefore also inhibits the activity of other methyltransferases ${ }^{[82]}$. Conversely, tazemetostat has been shown to be highly potent and selective for $\mathrm{EZH} 2^{[52]}$. Worth noting is the fact that combined treatment of specific $\mathrm{EZH} 2$ inhibitors either with differentiating agents, other epigenetic agents or chemotherapeutics resulted in enhanced anti-tumour activity in many STS studies. A number of STS are predominantly associated with paediatric onset and thus strategic combination of EZH2 targeted inhibitors with standard of care therapies will likely be the most 
Table 2. A summary table of the STS highlighted in this review and the effects of EZH2 modulation for each

\begin{tabular}{|c|c|c|c|}
\hline STS subtype & Drug tested & Anti-tumour effect? & RNAi phenotype \\
\hline \multirow[t]{3}{*}{ ERMS } & DZNep & Yes & Differentiation \\
\hline & GSK126 & Yes; with TPA & \\
\hline & MC1945/1948 & Yes & \\
\hline \multirow[t]{4}{*}{ ARMS } & DZNep & Yes & Proliferation inhibition, initiation of apoptosis \\
\hline & Tazemetostat & No & \\
\hline & MC1945/1948 & Yes & \\
\hline & Hybrid EZH2i+HDACi & Yes & \\
\hline \multirow[t]{5}{*}{ MRT } & DZNep & Yes; with etoposide, 5-Aza-CdR, SAHA & Reduced growth, SMARCB1 recovery \\
\hline & Tazemetostat & Yes (if INI1 deficient) & \\
\hline & GSK126 & Modest; with $\mathrm{HDACi}$ & \\
\hline & GSK343 & Modest & \\
\hline & UNC1999 & Modest & \\
\hline \multirow[t]{2}{*}{ ATRT } & DZNep & Yes (in-vitro only) & Proliferation reduction, initiation of apoptosis \\
\hline & GSK126 & Yes; with JQ1 & \\
\hline \multirow[t]{3}{*}{ Synovial sarcoma } & Tazemetostat & $\begin{array}{l}\text { Yes (if INI1 deficient) } \\
\text { Synergy with doxorubicin } \\
\text { Variable trial results }\end{array}$ & Proliferation inhibition \\
\hline & EPZ011989 & Yes & \\
\hline & EPZ005687 & Yes & \\
\hline Epitheliod sarcoma & Tazemetostat & Yes (if INI1 negative) & No data \\
\hline MPNST & DZNep & Yes & Growth inhibition, increased apoptosis \\
\hline Leiomyosarcoma & EPZ011989 & Yes; with dactolisib & No data \\
\hline \multirow[t]{2}{*}{ Liposarcoma } & GSK126 & Yes; with 5-Aza-dC & Proliferation inhibition, initiation of differentiation \\
\hline & GSK343 & Yes & \\
\hline
\end{tabular}

effective course of treatment for many STS.

\section{DECLARATIONS}

\section{Authors' contributions}

Made substantial contributions to conception and design of this review: Karolak M, Walters ZS

Original draft preparation: Karolak M, Walters ZS

Writing, review, and editing of manuscript: Karolak M, Tracy I, Shipley J, Walters ZS

Performed literature research: Karolak M

Preparation of figures: Tracy I, Walters ZS

\section{Availability of data and materials}

Not applicable.

\section{Financial support and sponsorship}

This work was supported by a Children with Cancer UK grant (Walters Z S) and by Sarcoma UK (Shipley J, Walters ZS). 


\section{Conflicts of interest}

All authors declare that there are no conflicts of interest.

\section{Ethical approval and consent to participate}

Not applicable.

\section{Consent for publication}

Not applicable.

\section{Copyright}

(c) The Author(s) 2021.

\section{REFERENCES}

1. Marchesi I, Fiorentino FP, Rizzolio F, Giordano A, Bagella L. The ablation of EZH2 uncovers its crucial role in rhabdomyosarcoma formation. Cell Cycle 2012;11:3828-36. DOI PubMed PMC

2. Vella S, Pomella S, Leoncini PP, et al. MicroRNA-101 is repressed by EZH2 and its restoration inhibits tumorigenic features in embryonal rhabdomyosarcoma. Clin Epigenetics 2015;7:82. DOI PubMed PMC

3. Shen JK, Cote GM, Gao Y, et al. Targeting EZH2-mediated methylation of H3K27 inhibits proliferation and migration of Synovial Sarcoma in vitro. Sci Rep 2016;6:25239. DOI PubMed PMC

4. Kawano S, Grassian AR, Tsuda M, et al. Preclinical Evidence of Anti-Tumor Activity Induced by EZH2 Inhibition in Human Models of Synovial Sarcoma. PLoS ONE 2016;11:e0158888. DOI PubMed PMC

5. Zhang P, Garnett J, Creighton CJ, et al. EZH2-miR-30d-KPNB1 pathway regulates malignant peripheral nerve sheath tumour cell survival and tumourigenesis. J Pathol 2014;232:308-18. DOI PubMed PMC

6. Knutson SK, Warholic NM, Wigle TJ, et al. Durable tumor regression in genetically altered malignant rhabdoid tumors by inhibition of methyltransferase EZH2. Proc Natl Acad Sci US A 2013;110:7922-7. DOI PubMed PMC

7. Moreno N, Kerl K. Preclinical evaluation of combined targeted approaches in malignant rhabdoid tumors. Anticancer Res 2016;36:3883-7. PubMed

8. Sun X, Guo W, Shen JK, Mankin HJ, Hornicek FJ, Duan Z. Rhabdomyosarcoma: Advances in Molecular and Cellular Biology. Sarcoma 2015;2015:232010. DOI PubMed PMC

9. Shi Y, Wang XX, Zhuang YW, Jiang Y, Melcher K, Xu HE. Structure of the PRC2 complex and application to drug discovery. Acta Pharmacol Sin 2017;38:963-76. DOI PubMed PMC

10. Ciarapica R, Miele L, Giordano A, Locatelli F, Rota R. Enhancer of zeste homolog 2 (EZH2) in pediatric soft tissue sarcomas: first implications. BMC Med 2011;9:63. DOI PubMed PMC

11. Ezhkova E, Pasolli HA, Parker JS, et al. Ezh2 orchestrates gene expression for the stepwise differentiation of tissue-specific stem cells. Cell 2009;136:1122-35. DOI PubMed PMC

12. Pereira JD, Sansom SN, Smith J, Dobenecker MW, Tarakhovsky A, Livesey FJ. Ezh2, the histone methyltransferase of PRC2, regulates the balance between self-renewal and differentiation in the cerebral cortex. Proc Natl Acad Sci U S A 2010;107:15957-62. DOI PubMed PMC

13. Zhang Q, Han Q, Zi J, et al. Mutations in EZH2 are associated with poor prognosis for patients with myeloid neoplasms. Genes Dis 2019;6:276-81. DOI PubMed PMC

14. Morschhauser F, Tilly H, Chaidos A, et al. Phase 2 Multicenter Study of Tazemetostat, an EZH2 Inhibitor, in Patients with Relapsed or Refractory Follicular Lymphoma. Blood 2019;134:123. DOI

15. Je EM, An CH, Yoo NJ, Lee SH. Mutational analysis of PIK3CA, JAK2, BRAF, FOXL2, IDH1, AKT1 and EZH2 oncogenes in sarcomas. APMIS 2012;120:635-9. DOI PubMed

16. Deng P, Chen QM, Hong C, Wang CY. Histone methyltransferases and demethylases: regulators in balancing osteogenic and adipogenic differentiation of mesenchymal stem cells. Int J Oral Sci 2015;7:197-204. DOI PubMed PMC

17. Dudakovic A, Camilleri ET, Xu F, et al. Epigenetic Control of Skeletal Development by the Histone Methyltransferase Ezh2. J Biol Chem 2015;290:27604-17. DOI PubMed PMC

18. Hemming S, Cakouros D, Isenmann S, et al. EZH2 and KDM6A act as an epigenetic switch to regulate mesenchymal stem cell lineage specification. Stem Cells 2014;32:802-15. DOI PubMed

19. Yu Y, Deng P, Yu B, et al. Inhibition of EZH2 Promotes Human Embryonic Stem Cell Differentiation into Mesoderm by Reducing H3K27me3. Stem Cell Reports 2017;9:752-61. DOI PubMed PMC

20. Zhang N, Zeng Z, Li S, Wang F, Huang P. High expression of EZH2 as a marker for the differential diagnosis of malignant and benign myogenic tumors. Sci Rep 2018;8:12331. DOI PubMed PMC

21. Wilson BG, Wang X, Shen X, et al. Epigenetic antagonism between polycomb and SWI/SNF complexes during oncogenic transformation. Cancer Cell 2010;18:316-28. DOI PubMed PMC

22. Kadoch C, Copeland RA, Keilhack H. PRC2 and SWI/SNF Chromatin Remodeling Complexes in Health and Disease. Biochemistry 2016;55:1600-14. DOI PubMed 
23. Bracken AP, Brien GL, Verrijzer CP. Dangerous liaisons: interplay between SWI/SNF, NuRD, and Polycomb in chromatin regulation and cancer. Genes Dev 2019;33:936-59. DOI PubMed PMC

24. Italiano A. Role of the EZH2 histone methyltransferase as a therapeutic target in cancer. Pharmacol Ther 2016;165:26-31. DOI PubMed

25. Yamagishi M, Uchimaru K. Targeting EZH2 in cancer therapy. Curr Opin Oncol 2017;29:375-81. DOI PubMed

26. Walters ZS, Villarejo-Balcells B, Olmos D, et al. JARID2 is a direct target of the PAX3-FOXO1 fusion protein and inhibits myogenic differentiation of rhabdomyosarcoma cells. Oncogene 2014;33:1148-57. DOI PubMed PMC

27. Ciarapica R, Russo G, Verginelli F, et al. Deregulated expression of miR-26a and Ezh2 in rhabdomyosarcoma. Cell Cycle 2009;8:1725. DOI PubMed

28. Changchien YC, Tátrai P, Papp G, et al. Poorly differentiated synovial sarcoma is associated with high expression of enhancer of zeste homologue 2 (EZH2). J Transl Med 2012;10:216. DOI PubMed PMC

29. Yamaga K, Osaki M, Kidani K, Shomori K, Yoshida H, Ito H. High expression of enhancer of zeste homologue 2 indicates poor prognosis in patients with soft tissue sarcomas. Mol Med Rep 2008;1:633-9. DOI PubMed

30. Yalçınkaya U, Uğraş N, Özgün G, et al. Enhancer of zeste homologue 2 (EZH2) expression in synovial sarcomas as a promising indicator of prognosis. Bosn J Basic Med Sci 2017;17:302-8. DOI PubMed PMC

31. Ramaglia M, D'Angelo V, Iannotta A, et al. High EZH2 expression is correlated to metastatic disease in pediatric soft tissue sarcomas. Cancer Cell Int 2016;16:59. DOI PubMed PMC

32. Cho YJ, Kim SH, Kim EK, et al. Prognostic implications of polycomb proteins ezh2, suz12, and eed1 and histone modification by H3K27me3 in sarcoma. BMC Cancer 2018;18:158. DOI PubMed PMC

33. Ciarapica R, Pezzullo M, Verginelli F, et al. Ezh2 is up-regulated and correlates with Ki67 and CD31 expression in human pediatric rhabdomyosarcoma. Published April 2010. DOI

34. . Wang, Q., Shapiro, K., Lu, Y., Shroyer, K. \& Hwang, S. EZH2 expression in rhabdomyosarcoma: A potential biomarker and therapeutic target. Modern Pathology 32 (2019).

35. Ciarapica R, Carcarino E, Adesso L, et al. Pharmacological inhibition of EZH2 as a promising differentiation therapy in embryonal RMS. BMC Cancer 2014;14:139. DOI PubMed PMC

36. Oh TJ, Adhikari A, Mohamad T, Althobaiti A, Davie J. TBX3 represses TBX2 under the control of the PRC2 complex in skeletal muscle and rhabdomyosarcoma. Oncogenesis 2019;8:27. DOI PubMed PMC

37. Ciarapica R, De Salvo M, Carcarino E, et al. The Polycomb group (PcG) protein EZH2 supports the survival of PAX3-FOXO1 alveolar rhabdomyosarcoma by repressing FBXO32 (Atrogin1/MAFbx). Oncogene 2014;33:4173-84. DOI PubMed

38. Rota R, Carcarino E, De Salvo M, et al. 453: Enhancer of Zeste Homolog 2 (EZH2) modulation in either embryonal or PAX3-FOXO1 alveolar rhabdomyosarcoma shows different anti-tumoral effects. European Journal of Cancer 2014;50:S109-10. DOI

39. Alimova I, Birks DK, Harris PS, et al. Inhibition of EZH2 suppresses self-renewal and induces radiation sensitivity in atypical rhabdoid teratoid tumor cells. Neuro Oncol 2013;15:149-60. DOI PubMed PMC

40. Popovski D, et al. Targeted catalytic inhibition of EZH2 synergizes with low-dose panobinostat in malignant rhabdoid tumor. NeuroOncology 19(2017). Published July 201741. DOI

41. Constantinidou, A. , et al. Epigenetic modulation in well differentiated (WD) and dedifferentiated (DD) liposarcoma (LPS): A novel therapeutic approach. Annals of Oncology 27(2016). DOI

42. Gounder M, Schöffski P, Jones RL, et al. Tazemetostat in advanced epithelioid sarcoma with loss of INI1/SMARCB1: an international, open-label, phase 2 basket study. Lancet Oncol 2020;21:1423-32. DOI PubMed

43. Italiano A, Soria J, Toulmonde M, et al. Tazemetostat, an EZH2 inhibitor, in relapsed or refractory B-cell non-Hodgkin lymphoma and advanced solid tumours: a first-in-human, open-label, phase 1 study. Lancet Oncol 2018;19:649-59. DOI PubMed

44. Morschhauser F, Tilly H, Chaidos A, et al. Tazemetostat for patients with relapsed or refractory follicular lymphoma: an open-label, single-arm, multicentre, phase 2 trial. Lancet Oncol 2020;21:1433-42. DOI PubMed

45. Yap TA, Winter JN, Giulino-Roth L, et al. Phase I Study of the Novel Enhancer of Zeste Homolog 2 (EZH2) Inhibitor GSK2816126 in Patients with Advanced Hematologic and Solid Tumors. Clin Cancer Res 2019;25:7331-9. DOI PubMed PMC

46. Jones BA, Varambally S, Arend RC. Histone Methyltransferase EZH2: A Therapeutic Target for Ovarian Cancer. Mol Cancer Ther 2018;17:591-602. DOI PubMed PMC

47. Schoffski P, Agulnik M, Stacchiotti S, et al. Phase 2 multicenter study of the EZH2 inhibitor tazemetostat in adults with synovial sarcoma (NCT02601950). JCO 2017;35:11057.

48. Marchesi I, Sanna L, Fais M, Fiorentino FP, Giordano A, Bagella L. 12-O-tetradecanoylphorbol-13-acetate and EZH2 inhibition: A novel approach for promoting myogenic differentiation in embryonal rhabdomyosarcoma cells. J Cell Physiol 2018;233:2360-5. DOI PubMed

49. Wang C. Childhood rhabdomyosarcoma: recent advances and prospective views. J Dent Res 2012;91:341-50. DOI PubMed PMC

50. Romanelli A, Stazi G, Fioravanti R, et al. Design of First-in-Class Dual EZH2/HDAC Inhibitor: Biochemical Activity and Biological Evaluation in Cancer Cells. ACS Med Chem Lett 2020;11:977-83. DOI PubMed PMC

51. Dawson LE, D'Agostino L, Hakim AA, et al. Induction of Myogenic Differentiation Improves Chemosensitivity of Chemoresistant Cells in Soft-Tissue Sarcoma Cell Lines. Sarcoma 2020;2020:8647981. DOI PubMed PMC

52. Kurmasheva RT, Sammons M, Favours E, et al. Initial testing (stage 1) of tazemetostat (EPZ-6438), a novel EZH2 inhibitor, by the Pediatric Preclinical Testing Program. Pediatr Blood Cancer 2017;64:e26218. DOI PubMed PMC

53. Unland R, Borchardt C, Clemens D, Kool M, Dirksen U, Frühwald MC. Analysis of the antiproliferative effects of 3-deazaneoplanocin A in combination with standard anticancer agents in rhabdoid tumor cell lines. Anticancer Drugs 2015;26:301-11. DOI PubMed 
54. Grinshtein N, Rioseco CC, Marcellus R, et al. Small molecule epigenetic screen identifies novel EZH2 and HDAC inhibitors that target glioblastoma brain tumor-initiating cells. Oncotarget 2016;7:59360-76. DOI PubMed PMC

55. Harding T, Swanson J, Van Ness B. EZH2 inhibitors sensitize myeloma cell lines to panobinostat resulting in unique combinatorial transcriptomic changes. Oncotarget 2018;9:21930-42. DOI PubMed PMC

56. Huang JP, Ling K. EZH2 and histone deacetylase inhibitors induce apoptosis in triple negative breast cancer cells by differentially increasing $\mathrm{H} 3 \mathrm{Lys}^{27}$ acetylation in the. BIM ;14:5735-42. DOI PubMed PMC

57. Takashina T, Kinoshita I, Kikuchi J, et al. Combined inhibition of EZH2 and histone deacetylases as a potential epigenetic therapy for non-small-cell lung cancer cells. Cancer Sci 2016;107:955-62. DOI PubMed PMC

58. Lindsay H, Kogiso M, Qi L, et al. AT-01 Therapeutic targeting of INI1 deficiency in pediatric atrt: A pre-clinical study utilizing patient derived orthotopic xenograft (PDOX) models. Neuro-Oncology 2015; ; 17:iii1.

59. Zhang A, Piunti A, Ozark P, et al. ATRT-02 Therapeutic targeting of EZH2 and BET BRD4 in AT/RT. Neuro-Oncology 2019;21:ii63.

60. Stacchiotti S, Schoffski P, Jones R, et al. Safety and efficacy of tazemetostat, a first-in-class EZH2 inhibitor, in patients (pts) with epithelioid sarcoma (ES) (NCT02601950). JCO 2019;37:11003.

61. Stacchiotti S, Zuco V, Tortoreto M, et al. Comparative Assessment of Antitumor Effects and Autophagy Induction as a Resistance Mechanism by Cytotoxics and EZH2 Inhibition in INI1-Negative Epithelioid Sarcoma Patient-Derived Xenograft. Cancers (Basel) 2019;11:1015. DOI PubMed PMC

62. Zhang P, Yang X, Ma X, et al. Antitumor effects of pharmacological EZH2 inhibition on malignant peripheral nerve sheath tumor through the miR-30a and KPNB1 pathway. Mol Cancer 2015;14:55. DOI PubMed PMC

63. Fourneaux B, Bourdon A, Dadone B, et al. Identifying and targeting cancer stem cells in leiomyosarcoma: prognostic impact and role to overcome secondary resistance to PI3K/mTOR inhibition. J Hematol Oncol 2019;12:11. DOI PubMed PMC

64. Velez AM, Okada T, Singer S. Aberrant expression of epigenetic modifiers in dedifferentiated liposarcoma (DDLS) and the effects of dual inhibition of DNA and histone methylation in DDLS cell lines. Abstract Book: Society of Surgical Oncology 69th Annual Cancer Symposium. Ann Surg Oncol 2016:23,S37-8. DOI

65. Wen Y, Cai J, Hou Y, Huang Z, Wang Z. Role of EZH2 in cancer stem cells: from biological insight to a therapeutic target. Oncotarget 2017;8:37974-90. DOI PubMed PMC

66. Xu K, Wu ZJ, Groner AC, et al. EZH2 oncogenic activity in castration-resistant prostate cancer cells is Polycomb-independent. Science 2012;338:1465-9. DOI PubMed PMC

67. Lee ST, Li Z, Wu Z, et al. Context-specific regulation of NF-kB target gene expression by EZH2 in breast cancers. Mol Cell 2011;43:798-810. DOI PubMed

68. Jung HY, Jun S, Lee M, et al. PAF and EZH2 induce Wnt/ $\beta$-catenin signaling hyperactivation. Mol Cell 2013;52:193-205. DOI PubMed PMC

69. Shi B, Liang J, Yang X, et al. Integration of estrogen and Wnt signaling circuits by the polycomb group protein EZH2 in breast cancer cells. Mol Cell Biol 2007;27:5105-19. DOI PubMed PMC

70. Kim E, Kim M, Woo DH, et al. Phosphorylation of EZH2 activates STAT3 signaling via STAT3 methylation and promotes tumorigenicity of glioblastoma stem-like cells. Cancer Cell 2013;23:839-52. DOI PubMed PMC

71. Wang X, Brea LT, Yu J. Immune modulatory functions of EZH2 in the tumor microenvironment: implications in cancer immunotherapy. Am J Clin Exp Urol 2019;7:85-91. PubMed PMC

72. Raj S, Miller LD, Triozzi PL. Addressing the Adult Soft Tissue Sarcoma Microenvironment with Intratumoral Immunotherapy. Sarcoma 2018;2018:9305294. DOI PubMed PMC

73. Christofides A, Karantanos T, Bardhan K, Boussiotis VA. Epigenetic regulation of cancer biology and anti-tumor immunity by EZH2. Oncotarget 2016;7:85624-40. DOI PubMed PMC

74. Carlo V, Mocavini I, Di Croce L. Polycomb complexes in normal and malignant hematopoiesis. J Cell Biol 2019;218:55-69. DOI PubMed PMC

75. Karantanos T, Chistofides A, Barhdan K, Li L, Boussiotis VA. Regulation of T Cell Differentiation and Function by EZH2. Front Immunol 2016;7:172. DOI PubMed PMC

76. Stairiker CJ, Thomas GD, Salek-Ardakani S. EZH2 as a Regulator of CD8+ T Cell Fate and Function. Front Immunol 2020;11:593203. DOI PubMed PMC

77. Herviou L, Cavalli G, Cartron G, Klein B, Moreaux J. EZH2 in normal hematopoiesis and hematological malignancies. Oncotarget 2016;7:2284-96. DOI PubMed PMC

78. Li B, Chng WJ. EZH2 abnormalities in lymphoid malignancies: underlying mechanisms and therapeutic implications. J Hematol Oncol 2019;12:118. DOI PubMed PMC

79. Huang S, Wang Z, Zhou J, et al. EZH2 Inhibitor GSK126 Suppresses Antitumor Immunity by Driving Production of Myeloid-Derived Suppressor Cells. Cancer Res 2019;79:2009-20. DOI PubMed

80. Goswami S, Apostolou I, Zhang J, et al. Modulation of EZH2 expression in T cells improves efficacy of anti-CTLA-4 therapy. J Clin Invest 2018;128:3813-8. DOI PubMed PMC

81. Xiao G, Jin LL, Liu CQ, et al. EZH2 negatively regulates PD-L1 expression in hepatocellular carcinoma. J Immunother Cancer 2019;7:300. DOI PubMed PMC

82. Miranda TB, Cortez CC, Yoo CB, et al. DZNep is a global histone methylation inhibitor that reactivates developmental genes not silenced by DNA methylation. Mol Cancer Ther 2009;8:1579-88. DOI PubMed PMC 